\title{
CCA Proxy Re-Encryption without Bilinear Maps in the Standard Model
}

\author{
Toshihide Matsuda ${ }^{1}$, Ryo Nishimaki $^{2}$, and Keisuke Tanaka ${ }^{1}$ \\ ${ }^{1}$ Department of Mathematical and Computing Sciences, Tokyo Institute of Technology,W8-55, \\ 2-12-1 Ookayama Meguro-ku, Tokyo 152-8552, Japan \\ \{matsuda5, keisuke\}@is.titech.ac.jp \\ ${ }^{2}$ NTT Laboratories, 3-9-11 Midori-cho, Musashino-shi, Tokyo, 180-8585, Japan \\ nishimaki.ryo@lab.ntt.co.jp
}

\begin{abstract}
Proxy re-encryption (PRE) is a cryptographic application proposed by Blaze, Bleumer, and Strauss. It is an encryption system with a special property in which the semi-honest third party, the proxy, can re-encrypt ciphertexts for Alice into other ciphertexts for Bob without using Alice's secret key. We can classify PRE into bidirectional and unidirectional schemes. Canetti and Hohenberger formalized the semantic security under chosen ciphertext attack for PRE, the PRECCA security. Several schemes satisfy the PRE-CCA security as a bidirectional or unidirectional scheme. However, some PRE schemes need a bilinear map in the standard model, and the other PRE schemes are PRE-CCA secure in the random oracle model before our work. In this paper, we construct a bidirectional PRECCA proxy re-encryption without bilinear maps in the standard model. We study lossy trapdoor functions (LTDFs) based on the decisional Diffie-Hellman (DDH) assumption proposed by Peikert and Waters. We define a new variant of LTDFs, re-applicable LTDFs, which are specialized LTDFs for PRE, and use them for our scheme.
\end{abstract}

\section{Introduction}

\subsection{Background}

Proxy re-encryption (PRE) is a cryptographic application proposed by Blaze, Bleumer, and Strauss [4]. It is an encryption system with a special property in which the semihonest third party, the proxy, can re-encrypt ciphertexts for Alice into other ciphertexts for Bob without using Alice's secret key. In the other words, if the proxy has a reencryption key $r k_{A \leftrightarrow B}$ from Alice to Bob, the proxy can translate a ciphertext $C_{A}$ under Alice's public key $p k_{A}$ into another ciphertext $C_{B}$ under Bob's public key $p k_{B}$. This translation requires only $r k_{A \leftrightarrow B}$ and keeps the message $m$ secret for the proxy. There are many PRE cryptographic applications, such as email-forwarding, secure file systems, DRM, and secure mailing lists [2/4|1121].

Ivan and Dodis classified PRE into two types, bidirectional and unidirectional [10]. The former means that, if the proxy has the re-encryption key $r k_{A \leftrightarrow B}$, the proxy cannot only re-encrypt ciphertexts from Alice to Bob, but also in the opposite direction. That is, we can assume that the re-encryption key $r k_{A \leftrightarrow B}$ from Alice to Bob is identical to 
$r k_{B \leftrightarrow A}$ from Bob to Alice. On the other hand, the latter means that, the re-encryption key $r k_{A \rightarrow B}$ from Alice to Bob never helps re-encryption of the opposite direction. They also considered the notions of single-hop and multi-hop. The former means that a reencrypted ciphertext cannot be further re-encrypted. In contrast, the later means that a ciphertext can be re-encrypted from Alice to Bob to Carol and so on. We discuss only bidirectional schemes in this paper.

The PRE-CCA Security. The security notion of indistinguishability against chosen cipher text attacks (IND-CCA) on encryption systems was proposed by Naor and Yung [15]. Many IND-CCA secure encryption systems have been proposed.

Canetti and Hohenberger applied the notion of IND-CCA to PRE for the bidirectional scheme [6]. They formalized the security notion as the (bidirectional) PRE-CCA security. They also investigated simulation-based security definitions that guarantee universally composable security. They constructed a bidirectional and multi-hop PRECCA scheme based on the decisional bilinear Diffie-Hellman (DBDH) assumption, which requires bilinear maps. Later, some research groups proposed bidirectional or unidirectional PRE-CCA schemes with bilinear maps or in the random oracle model [7|12|20|22].

\subsection{Our Contribution}

We construct a bidirectional and multi-hop PRE-CCA scheme without bilinear maps in the standard model. All previous PRE-CCA secure schemes in the standard model use bilinear maps. Our scheme is constructed in three steps.

First, we define a new cryptographic primitive, re-applicable lossy trapdoor functions (re-applicable LTDFs), which are specialized lossy trapdoor functions for PRE. They consist of nine algorithms and a set of tags, (ParGen, LossyGen, LossyEval, Lossylnv, Relndex, ReEval, PrivReEval, Trans, FakeKey) and $\mathcal{T}$. Second, we construct a bidirectional PRE-CCA scheme by using re-applicable LTDFs. We modify the original Peikert and Waters encryption scheme on several points. Third, we construct re-applicable LTDFs based on the decisional Diffie-Hellman (DDH) assumption.

Our Techniques. As stated above, we modify the original Peikert and Waters encryption scheme on several points for PRE. Our PRE scheme uses an index of all-but-one functions as the public parameter. The original scheme uses this index as a part of a public key. Our scheme generates a signature of a part of a ciphertext $\left(c_{2}, c_{3}\right)$ in the encryption scheme. The original scheme generates a signature of all the main parts of a ciphertext $\left(c_{1}, c_{2}, c_{3}\right)$ in the encryption process. The most different point is that our scheme re-encrypts $c_{1}$ from $p k_{A}$ to $p k_{B}$ by using $r k_{A \leftrightarrow B}$.

We modify LTDFs proposed by Peikert and Waters on one point for construction of re-applicable LTDFs. It is that an injective index is not $\operatorname{Enc}_{p k}(\boldsymbol{I})$, but $\mathrm{Enc}_{p k}(\tau \boldsymbol{I})$, where $\tau$ is a tag and $\boldsymbol{I}$ is the identity matrix. This modification is a technical change that satisfies the definition of re-applicable LTDFs.

The Peikert and Waters Encryption. Peikert and Waters proposed LTDFs and constructed an IND-CCA public-key encryption by using LTDFs [17]. We briefly review their encryption scheme. Let $f_{s}$ and $f_{s^{\prime}}$ be functions with a domain $\{0,1\}^{n}$, where $f_{s}$ is 
an injective function with a trapdoor $t d$, and $f_{s^{\prime}}$ is a lossy function of which the size of a range is $2^{n-k}$ at most. LTDFs have the following property: Given $f$, the distinguisher cannot distinguish whether $f$ is $f_{s}$ or $f_{s^{\prime}}$. They constructed them as $f_{s}(\boldsymbol{x})=\boldsymbol{x} \operatorname{Enc}_{p k}(\boldsymbol{I})$ and $f_{s^{\prime}}(\boldsymbol{x})=\boldsymbol{x} \mathrm{Enc}_{p k}(\mathbf{0})$ where $\boldsymbol{I}$ and $\mathbf{0}$ is the identity and the zero matrix, and Enc is a homomorphic matrix encryption. From the homomorphism of Enc, we obtain $f_{s}(\boldsymbol{x})=$ $\boldsymbol{x} \mathrm{Enc}_{p k}(\boldsymbol{I})=\mathrm{Enc}_{p k}(\boldsymbol{x} \boldsymbol{I})=\mathrm{Enc}_{p k}(\boldsymbol{x})$ and $f_{s^{\prime}}(\boldsymbol{x})=\boldsymbol{x} \mathrm{Enc}_{p k}(\mathbf{0})=\mathrm{Enc}_{p k}(\boldsymbol{x} \mathbf{0})=\mathrm{Enc}_{p k}(\mathbf{0})$. We can reconstruct $\boldsymbol{x}$ from $f_{s}(\boldsymbol{x})$ with a secret key $s k$. On the other hand, we never obtain $\boldsymbol{x}$ from $f_{s^{\prime}}(\boldsymbol{x})$ information-theoretically. They also proposed all-but-one trapdoor functions which have similar property to LTDFs. Let $g_{s^{\prime}, b^{*}}$ be a function with a domain $B \times\{0,1\}^{n}$, where $B$ is a finite set and $b^{*} \in B$. For every $b \neq b^{*}, g_{s^{\prime}, b^{*}}(b, \cdot)$ is an invertible function with a trapdoor $t d$. On the other hand, $g_{s^{\prime}, b^{*}}\left(b^{*}, \cdot\right)$ is a lossy function.

Peikert and Waters constructed their IND-CCA encryption scheme by using $f_{s}$ and $g_{s^{\prime}, b^{*}}$ as follows. The encryption algorithm randomly chooses $x \in\{0,1\}^{n}$ and selects a key pair of a one-time signature $\left(v k, s k_{\sigma}\right)$. Then, it computes a ciphertext as

$$
C=\left(v k, c_{1}, c_{2}, c_{3}, \sigma\right)=\left(v k, f_{s}(x), g_{s^{\prime}, b^{*}}(v k, x), h(x) \oplus m, \operatorname{SigSign}\left(s k_{\sigma}, c_{1}, c_{2}, c_{3}\right)\right),
$$

where $h$ is a pair-wise independent hash and SigSign is a signing algorithm in a signature scheme. They proved that this scheme satisfied the IND-CCA security.

Observation of the Peikert and Waters Encryption: Free Part for Signature. The above encryption algorithm must sign all $\left(c_{1}, c_{2}, c_{3}\right)$ and make the signature $\sigma$. The signature $\sigma$ and $v k$ guarantees that $\left(c_{1}, c_{2}, c_{3}\right)$ is signed with the signing key $s k_{\sigma}$. That is, $\left(c_{1}, c_{2}, c_{3}\right)$ is fixed by $\sigma$ and $v k$. For this fixed $\left(c_{1}, c_{2}, c_{3}\right)$, the Peikert and Waters encryption achieves the IND-CCA security.

However, we find that it is not necessary to sign all $\left(c_{1}, c_{2}, c_{3}\right)$ in order to achieve the IND-CCA security. Moreover, we find that it does not need to sign $c_{1}$. The reason is as follows. If $\left(c_{1}, c_{2}, c_{3}\right)$ is fixed by $\sigma$ and $v k$, randomness $x$ and a message $m$ also are fixed because of the injectivity of $f_{s}$ and $g_{s^{\prime}, b^{*}}$. That is, we can consider $\sigma$ as a signature of $x$ and $m$ as well as $\left(c_{1}, c_{2}, c_{3}\right)$. In addition, if $x$ and $m$ are determined, $\left(c_{1}, c_{2}, c_{3}\right)$ is determined. We understand that it is necessary to sign $x$ and $m$, not $\left(c_{1}, c_{2}, c_{3}\right)$. We replace a signature of $\left(c_{1}, c_{2}, c_{3}\right)$ with a signature of $\left(c_{2}, c_{3}\right)$. A pair of $x$ and $m$ is fixed similarly to the case of $\left(c_{1}, c_{2}, c_{3}\right)$ because of the injectivity of $g_{s^{\prime}, b^{*}}$. That is, the signature of $\left(c_{2}, c_{3}\right)$ performs tasks of a signature of $\left(c_{1}, c_{2}, c_{3}\right)$. We do not need the signature of $c_{1}$ to achieve the IND-CCA security. This free $c_{1}$ is very important in constructing our proposed scheme, which satisfies the bidirectional PRE-CCA security.

Remarks on Our Scheme. We discuss two points of our scheme: 1. Comparison of efficiency and 2. Construction based on other assumptions.

Our scheme uses an index of re-applicable LTDFs as a public key. We represent this key as an $n \times n$ matrix, which has $n^{2}$ group elements. The public parameter contains $n \times n$ matrix as well as public keys since all-but-one trapdoor functions are based on the DDH assumption. One ciphertext and one re-encryption key have $O(n)$ group elements. However, in most of the previous schemes, they consist of a constant number of group elements. For example, one public key is one group element, and one ciphertext consists of five group elements, a verification key, and a signature in the Canetti and Hohenberger's bidirectional scheme, which satisfies the PRE-CCA security as well as 
ours [6]. Time complexity as well as space complexity are larger than others. Therefore, future work is to construct more efficient schemes.

LTDFs are constructed from various assumptions. Therefore, one may think that we can construct PRE-CCA secure schemes from other assumptions. However, we do not know how to construct PRE-CCA secure schemes from other assumptions now. Factoring, quadratic residue, RSA, or Paillier-based LTDFs do not clearly satisfy the definition of re-applicable lossy trapdoor functions. One might think that decision linear or lattice-based LTDFs work in our proposal, but this is not clear. In other words, they do not guarantee several properties of re-applicable LTDFs. However, we might be able to use LTDFs based on them with other techniques for PRE.

\subsection{Related Work}

We review previous work on PRE and LTDFs.

Proxy Re-Encryptions. Mambo and Okamoto first proposed the concept of proxy encryption, which delegates the ability of decryption through an interaction [13]. Based on their concept, Blaze, Bleumer, and Strauss proposed the notion of proxy cryptography. PRE is one concept in proxy cryptography [4]. Their construction is nearly similar to the ElGamal encryption and satisfies the CPA security. A re-encryption key is made from the division of secret keys in the scheme. Later, Ivan and Dodis point out that Blaze et.al.'s scheme is bidirectional and multi-hop. Ateniese, Fu, Green, and Hohenberger proposed the first unidirectional scheme, which was single-hop and satisfied the CPA security with a bilinear map [2]. Deng, Weng, Liu, and Chen constructed a bidirectional and single-hop PRE scheme without a bilinear map in the random oracle model [7]. Libert and Vergnaud discussed the unidirectional PRE-CCA security and constructed an unidirectional and single-hop PRE-RCCA 1 scheme with a bilinear map [12]. Shao and Cao proposed an unidirectional and single-hop PRE-CCA scheme in the random oracle model [20]2. Weng, Chow, Yang, and Deng improved Shao and Cao's scheme, and their scheme also is in the random oracle model [22]. We put this previous work in chronological order in Figure 1. ROM stands for the random oracle model.

Hohenberger, Rothblum, shelat, and Vaikuntanathan argued the existence of obfuscators with PRE and practically constructed an obfuscator as a PRE scheme [9]. Their scheme is CPA-secure.

Recently, Ateniese, Benson, and Hohenberger introduced an additional property on PRE, which is key-privacy (or anonymous) [1]. It is desirable to have this property, if the proxy can freely re-encrypt ciphertexts. Our scheme does not have the key-private property. They constructed a key-private scheme with bilinear maps in the standard model, which satisfies the PRE-CPA security, not CCA.

\footnotetext{
${ }^{1}$ This security notion is truly weaker than the CCA security and stronger than the CPA security. An adversary can use a decryption oracle in a restricted way. If he sends messages $\left(m_{0}, m_{1}\right)$ to the challenger and obtains a challenge $c$, the adversary cannot query ciphertexts of challenge messages $m_{0}$ and $m_{1}$ to the decryption oracle.

${ }^{2}$ Two research groups posed questions on the security model of this paper and published their discussions on ePrint Archive [22[23]. However, they do not effect our results and we do not mention this in this paper.
} 


\begin{tabular}{|c|c|c|c|c|c|c|}
\hline Authors & Direction & Hop & Assumption & Security & Bilinear Map & ROM \\
\hline Blaze et.al. [4] & $\leftrightarrow$ & multi & DDH on $\mathbb{G}_{p}$ & PRE-CPA & no & no \\
\hline Ateniese et.al. [2] & $\rightarrow$ & single & eDBDH & PRE-CPA & yes & no \\
\hline Canetti and Hohenberger [6] & $\leftrightarrow$ & multi & DBDH & PRE-CCA & yes & no \\
\hline Libert and Vergnaud [12] & $\rightarrow$ & single & 3-wDBDHI & PRE-RCCA & yes & no \\
\hline Deng et.al. [7] & $\leftrightarrow$ & single & mCDH on $\mathbb{G}_{p}$ & PRE-CCA & no & yes \\
\hline Shao and Cao [20] & $\rightarrow$ & single & DDH on $\mathbb{Z}_{N^{2}}$ & PRE-CCA & no & yes \\
\hline Weng et.al. [22] & $\rightarrow$ & single & CDH on $\mathbb{G}_{p}$ & PRE-CCA & no & yes \\
\hline This paper & $\leftrightarrow$ & multi & DDH on $\mathbb{G}_{p}$ & PRE-CCA & no & no \\
\hline
\end{tabular}

Fig. 1. Comparison of our work with previous work

Lossy Trapdoor Functions. We review previous work related to LTDFs. Peikert and Waters proposed notions of LTDFs [17]. They showed cryptographic applications based on LTDFs, such as a (ordinary) trapdoor function, a collision-resistant hash function, an oblivious transfer, and an IND-CCA encryption scheme. They also showed the constructions of LTDFs based on the DDH assumption and the LWE assumption. They mentioned that the Paillier encryption realized LTDFs by the similar methodology to the construction based on the DDH assumption. Later, Rosen and Segev noted that the Damgård-Jurik encryption scheme simply satisfied the definition of LTDFs because of its number-theoretic property [18]. The Damgård-Jurik encryption scheme is considered as a generalized Paillier encryption. In addition to [18], Freedman, Goldreich, Kiltz, Rosen, and Segev proposed more constructions of LTDFs [8]. They are based on the $d$ Linear assumption and the QR assumption. Mol and Yilek showed that slightly LTDFs are sufficient for constructing a IND-CCA secure public-key encryption scheme [14]. Slightly LTDFs lost a $(1-\omega(\log n))$ fraction of all its input bits.

Other applications of LTDFs have been proposed. Rosen and Segev proposed a new primitive, a one-way function under correlated products [19]. They showed the construction of a one-way function under correlated products from LTDFs and the INDCCA secure encryption by using this primitive. Boldyreva, Fehr, and O'Neill applied LTDFs to the construction of deterministic encryption [5]. They constructed a CCAsecure deterministic encryption scheme in the standard model, where the CCA security meant the sense of the semantic security on a message, not indistinguishability of messages. Bellare, Hofheinz, and Yilek formalized a new security notion of encryption, selective opening attack, which meant that it kept secret even if an adversary selectively obtained messages and randomness of ciphertexts [3]. They used LTDFs for the above purpose. Nishimaki, Fujisaki, and Tanaka used all-but-one trapdoor functions for the universally composable commitment scheme [16]. They first proposed a non-interactive string-commitment scheme, which is universally composable.

\section{Organization}

In Section 2, we show preliminaries to describe our scheme. In Section 3, we define reapplicable LTDFs. In Section 4 we review the definition of bidirectional PRE schemes, propose our scheme, and describe a sketch of a proof of it. In Section 5, we construct re-applicable LTDFs based on the DDH assumption. 


\section{Preliminaries}

In this section, we show preliminaries to describe our scheme.

\subsection{Notation}

Let $S$ be a finite set. $s \in_{R} S$ denotes that the element $s$ is chosen from $S$ uniformly at random. For probabilistic algorithm $A, y \leftarrow A(x)$ denotes that $A$ outputs $y$ on input $x$ with uniform randomness. If $A$ runs in time polynomial in the security parameter, then $A$ is a probabilistic polynomial-time (PPT) algorithm. We say that function $f: \mathbb{N} \rightarrow$ $[0,1]$ is negligible in $\lambda \in \mathbb{N}$ if for every constant $c \in \mathbb{N}$ there exists $k_{c} \in \mathbb{N}$ such that $f(\lambda)<\lambda^{-c}$ for any $\lambda>k_{c}$. We say that function $g: \mathbb{N} \rightarrow[0,1]$ is overwhelming in $\lambda \in \mathbb{N}$ if function $f(\lambda)=1-g(\lambda)$ is negligible in $\lambda \in \mathbb{N}$. Let $X_{\lambda}$ and $Y_{\lambda}$ denote random variables over a finite set $Z_{\lambda} \subset\{0,1\}^{\lambda}$, where $\lambda \in \mathbb{N}$ is the security parameter. We say that $X_{\lambda}$ and $Y_{\lambda}$ are (computationally) indistinguishable if, for every distinguisher $D$, $\left|\operatorname{Pr}\left[D\left(X_{\lambda}\right)=1\right]-\operatorname{Pr}\left[D\left(Y_{\lambda}\right)=1\right]\right|$ is negligible in $\lambda \in \mathbb{N}$. We say that $X_{\lambda}$ and $Y_{\lambda}$ are statistically indistinguishable if $\sum_{z \in Z_{\lambda}} \mid \operatorname{Pr}\left[X_{\lambda}=z\right]-\operatorname{Pr}\left[Y_{\lambda}=z\right]$ is negligible in $\lambda \in \mathbb{N}$.

\subsection{DDH Assumption}

We review the DDH assumption. Let $\mathcal{G}$ be an algorithm that takes as input a security parameter $\lambda$ and outputs a tuple ( $p, \mathbb{G}, g$ ), where $p$ is a prime with $2^{\lambda-1} \leq p<2^{\lambda}$, $\mathbb{G}$ is a cyclic group of prime order $p$, and $g$ is a generator of $\mathbb{G}$.

Assumption 1 (The Decisional Diffie-Hellman Assumption). For any PPT adversary $A$, the advantage $\operatorname{Adv}_{A}(k)$ is negligible in the security parameter $k$.

$$
\operatorname{Adv}_{A}(k)=\left|\operatorname{Pr}\left[A\left((p, \mathbb{G}, g), g^{a}, g^{b}, g^{a b}\right)=1\right]-\operatorname{Pr}\left[A\left((p, \mathbb{G}, g), g^{a}, g^{b}, g^{c}\right)=1\right]\right|
$$

The probability is over the random choices of $(p, \mathbb{G}, g) \leftarrow \mathcal{G}(\lambda)$, the random choices of $a, b, c \in \mathbb{Z}_{p}$ and the random coin of $A$.

\subsection{All-But-One Trapdoor Functions}

We review all-but-one trapdoor functions to describe our scheme. All-but-one trapdoor functions are made from the DDH assumption[17].

Definition 1 (All-but-one trapdoor functions). A collection of $(n, k)$-all-but-one trapdoor functions is a tuple of PPT algorithms $\left(\mathrm{G}_{\mathrm{abo}}, \mathrm{F}_{\mathrm{abo}}, \mathrm{F}_{\mathrm{abo}}^{-1}\right)$ and sequence of branch sets $B=\left\{B_{\lambda}\right\}$ such that:

All-but-one property: Given a lossy branch $b^{*} \in B_{\lambda}$, the algorithm $\mathrm{G}_{\mathrm{abo}}\left(1^{\lambda}, b^{*}\right)$ outputs a pair $(s, t d)$. For every $b \in B_{\lambda} \backslash\left\{b^{*}\right\}$, the algorithm $\mathrm{F}_{\mathrm{abo}}(s, b, \cdot)$ computes an injective function $f_{s, b}(\cdot)$ over $\{0,1\}^{n}$, and $\mathrm{F}_{\text {abo }}^{-1}(t d, b, \cdot)$ computes $f_{s, b}^{-1}(\cdot)$. For the lossy branch $b^{*}, \mathrm{~F}_{\text {abo }}\left(s, b^{*}, \cdot\right)$ computes a lossy function $f_{s, b^{*}}(\cdot)$ over $\{0,1\}^{n}$, where $\left|f_{s, b^{*}}\left(\{0,1\}^{n}\right)\right| \leq 2^{n-k}$.

Indistinguishability: For every $b_{1}^{*}$ and $b_{2}^{*} \in B_{\lambda}$, the first output $s_{0}$ of $\mathrm{G}_{\mathrm{abo}}\left(1^{\lambda}, b_{0}^{*}\right)$ and the first output $s_{1}$ of $\mathrm{G}_{\mathrm{abo}}\left(1^{\lambda}, b_{1}^{*}\right)$ are computationally indistinguishable. 


\section{Re-applicable Lossy Trapdoor Functions}

In this section, we propose a new primitive, re-applicable LTDFs, which is an extension of LTDFs. Peikert and Waters proposed LTDFs and all-but-one functions in STOC'08 [17].

For our purpose, we transform LTDFs in several points. First, we add one algorithm, the parameter-generation algorithm ParGen. This algorithm generates public parameters which is common to every algorithm and applied in every evaluation. We introduce ParGen since PRE schemes are used in the multi-user setting, not single-user setting. In addition, the validity checks of ciphertexts require that each ciphertext of the ElGamal encryption in an index of LTDFs has common randomness for every user.

Second, we modify LTDFs so that the function-generation algorithm receives a tag in a set of tags $\mathcal{T}$, not injective or lossy commands. For every tag $\tau$, except one special lossy tag $\tau_{\text {los }}$, the function-generation algorithm outputs an index that represents an injective function. On the other hand, the function-generation algorithm given $\tau_{\text {los }}$ outputs an index that represents a lossy function.

Third, we define five new algorithms Relndex, ReEval, PrivReEval, Trans, and FakeKey. Relndex, ReEval, PrivReEval, and Trans are deterministic, and FakeKey is probabilistic. We apply Relndex for generating re-encryption keys, ReEval for evaluation of re-encryption, and PrivReEval for a validity check of ciphertexts. The algorithms Trans and FakeKey are only used in the proof. The algorithm Trans guarantees the transitivity between re-encryption keys. In other word, we can make a re-encryption key $r k_{j \leftrightarrow k}$ from $r k_{i \leftrightarrow j}$ and $r k_{i \leftrightarrow k}$. The algorithm FakeKey generates a pair of public and re-encryption keys $\left(p k_{j}, r k_{i \leftrightarrow j}\right)$ from another public key $p k_{i}$. Moreover, even if $p k_{i}$ represents a lossy function, FakeKey always outputs $p k_{j}$, which represents an injective function. This property is necessary for the last modification in our proof. We introduce $\mathcal{T}$ to provide this property.

We call this new primitive, a collection of re-applicable LTDFs. They are specialized LTDFs for PRE. If we unify ParGen and LossyGen, ignore the other new algorithms, and define $\mathcal{T}=\left\{\tau_{\text {inj }}, \tau_{\text {los }}\right\}$, we can consider this new primitive as (ordinary) LTDFs proposed by Peikert and Waters.

Definition 2 (Re-applicable LTDFs with respect to function indices). Let (ParGen, LossyGen, LossyEval, Lossylnv, Relndex, ReEval, PrivReEval, Trans, FakeKey) be a tuple of PPT algorithms, and $\mathcal{T}$ be a set of tags that contains one lossy element $\tau_{\text {los }}$. The algorithm ParGen $\left(1^{\lambda}\right)$ outputs a public parameter par. The other algorithms apply the parameter par to their computations. Hereafter, we omit the input of the public parameter par for the algorithms.

A collection of re-applicable $(n, k)$-lossy trapdoor functions with respect to function indices is a tuple of the PPT algorithms (ParGen, LossyGen, LossyEval, Lossylnv, Relndex, ReEval, PrivReEval, Trans, FakeKey) such that:

Injectivity; For every public parameter par $\leftarrow$ ParGen $\left(1^{\lambda}\right)$ and every tag $\tau \in$ $\mathcal{T} \backslash\left\{\tau_{\text {los }}\right\}$, LossyGen $(\tau)$ outputs a pair of a function index and its trapdoor $(s, t d)$, LossyEval $(s, \cdot)$ computes an injective function $f_{s, \tau}(\cdot)$ over $\{0,1\}^{n}$, and Lossylnv $(t d, \tau, \cdot)$ computes $f_{s, \tau}^{-1}(\cdot)$. 
(We represent the function $f_{s, \tau}$, not $f_{s}$, in order to clarify a tag $\tau$. If we do not need to clarify a tag, we represent a function as $f_{s, \star}$ )

Lossiness: For every public parameter par $\leftarrow \operatorname{ParGen}\left(1^{\lambda}\right)$, the algorithm LossyGen $\left(\tau_{\text {los }}\right)$ outputs $(s, \perp)$ and LossyEval $(s, \cdot)$ computes a function $f_{s, \tau_{\text {los }}}(\cdot)$ over $\{0,1\}^{n}$, where $\left|f_{s, \tau_{\text {los }}}\left(\{0,1\}^{n}\right)\right| \leq 2^{n-k}$.

Indistinguishability between injective and lossy indices: Let $X_{\lambda}$ denote the distribution of (par, $\left.s_{\mathrm{inj}}, \tau\right)$, and let $Y_{\lambda}$ denote the distribution of (par, $s_{\mathrm{los}}, \tau^{\prime}$ ), where par is a public parameter from $\operatorname{ParGen}\left(1^{\lambda}\right), \tau$ and $\tau^{\prime}$ are random elements in $\mathcal{T}$, and the function indices $s_{\text {inj }}$ and $s_{\text {los }}$ are the first element outputs from LossyGen $(\tau)$ and LossyGen $\left(\tau_{\text {los }}\right)$. Then, $\left\{X_{\lambda}\right\}$ and $\left\{Y_{\lambda}\right\}$ are computationally indistinguishable.

Re-applying with respect to function indices: Let $\tau_{i}$ and $\tau_{j}$ be any tags with $\tau_{i} \neq \tau_{\text {los }}$ and $\tau_{j} \neq \tau_{\text {los. }}$. The algorithm Relndex $\left(t d_{i}, t d_{j}\right)$ outputs $s_{i \leftrightarrow j}$, where $t d_{i}$ and $t d_{j}$ are the second elements of LossyGen $\left(\tau_{i}\right)$ and LossyGen $\left(\tau_{j}\right)$. Then, for every $x \in\{0,1\}^{n}$, $x=\operatorname{LossyInv}\left(t d_{j}, \tau_{i}, \operatorname{ReEval}\left(s_{i \leftrightarrow j}, \operatorname{LossyEval}\left(s_{i}, x\right)\right)\right)$. We remark that Lossylnv takes $\tau_{i}$ as one of the inputs, not $\tau_{j}$.

Generating proper outputs: Let $c$ be an output from $\operatorname{ReEval}\left(s_{i \leftrightarrow j}, \operatorname{LossyEval}\left(s_{i}, x\right)\right)$, where $s_{i \leftrightarrow j}$ and $s_{i}$ have the same meaning as that in the above paragraph. Then, PrivReEval $\left(x, \tau_{i}, \tau_{j}, s_{j}\right)$ outputs the same $c$, where $x, \tau_{i}, \tau_{j}$, and $s_{j}$ have the same meaning as that in the above paragraph. That is, $\operatorname{ReEval}\left(s_{i \leftrightarrow j}, \operatorname{LossyEval}\left(s_{i}, \cdot\right)\right)$ and PrivReEval $\left(\cdot, \tau_{i}, \tau_{j}, s_{j}\right)$ are equivalent as a function (i.e. Any output of $\operatorname{ReEval}\left(s_{i \leftrightarrow j}\right.$, LossyEval $\left.\left(s_{i}, \cdot\right)\right)$ is independent of $s_{i}$.).

Transitivity: Let $\left(s_{i}, t d_{i}\right),\left(s_{j}, t d_{j}\right)$ and $\left(s_{k}, t d_{k}\right)$ be outputs from LossyGen $\left(\tau_{i}\right)$, $\operatorname{LossyGen}\left(\tau_{j}\right)$, and LossyGen $\left(\tau_{k}\right)$, and let $s_{i \leftrightarrow j}$ and $s_{i \leftrightarrow k}$ be outputs from Relndex $\left(t d_{i}, t d_{j}\right)$ and Relndex $\left(t d_{i}, t d_{k}\right)$, respectively. Then, Trans $\left(s_{i \leftrightarrow j}, s_{i \leftrightarrow k}\right)$ outputs $s_{j \leftrightarrow k}$ which is the same output from Relndex $\left(t d_{j}, t d_{k}\right)$.

Statistical indistinguishability of the fake key: The algorithm FakeKey $\left(s_{i}, \tau_{i}\right)$ outputs $\left(s_{j}^{\prime}, s_{i \leftrightarrow j}^{\prime}, \tau_{j}^{\prime}\right)$, where $s_{i}$ is the first element of an output from LossyGen $\left(\tau_{i}\right)$. Let $X_{\lambda}$ denote the distribution of ( $p a r, s_{i}, s_{j}, s_{i \leftrightarrow j}, \tau_{i}, \tau_{j}$ ), and let $Y_{\lambda}$ denote the distribution of (par, $s_{i}, s_{j}^{\prime}, s_{i \leftrightarrow j}^{\prime}, \tau_{i}, \tau_{j}^{\prime}$ ), where each par, $s_{j}, s_{i \leftrightarrow j}$, and $\tau_{j}$ has the same meaning as that in the above paragraph. Then, $\left\{X_{\lambda}\right\}$ and $\left\{Y_{\lambda}\right\}$ are statistically indistinguishable.

Generation of injective functions from lossy functions: Let $s$ be the first element of an output from FakeKey $\left(s_{\text {los }}, \tau\right)$, where $\tau$ is a tag and $s_{\text {los }}$ is the first element of an output from LossyGen $\left(\tau_{\text {los }}\right)$. Then, for every $\tau$, LossyEval $(s, \cdot)$ represents an injective function $f_{s, \star}$ with overwhelming probability, where a random variable is the randomness of FakeKey $\left(s_{\mathrm{los}}, \tau\right)$. (We do not require other properties of index $s$ if $f_{s, \star}$ is injective. The function $f_{s, \star}$ cannot have any trapdoor information.)

\section{Bidirectional and Multi-Hop PRE-CCA Scheme}

In this section, we first review the definition of a bidirectional PRE scheme. Then, we describe our scheme and show a sketch of the proof.

A bidirectional PRE scheme consists of six algorithms $\Pi=$ (Setup, KeyGen, Enc, Dec, ReKeyGen, and ReEnc) as follows. $P P \leftarrow \operatorname{Setup}\left(1^{\lambda}\right)$ : Given a security parameter $1^{\lambda}$, the setup algorithm outputs a public parameter $P P$. This algorithm is executed by 
a trusted third party. $(p k, s k) \leftarrow \operatorname{KeyGen}(P P)$ : Given a public parameter $P P$, the key generation algorithm outputs a public key $p k$ and a secret key $s k . C \leftarrow \operatorname{Enc}(P P, p k, m)$ : Given a public key $p k$ and a message $m \in \mathcal{M}$, the encryption algorithm outputs a ciphertext $C$, where $\mathcal{M}$ is a message space. $r k_{i \leftrightarrow j} \leftarrow \operatorname{ReKeyGen}\left(P P, s k_{i}, s k_{j}\right)$ : Given a pair of secret keys $s k_{i}, s k_{j}$, where $i \neq j$, this algorithm outputs a re-encryption key $r k_{i \leftrightarrow j}$. We call $r k_{i \leftrightarrow j}$ the re-encryption key between $i$ and $j . C_{j} \leftarrow \operatorname{ReEnc}\left(P P, r k_{i \leftrightarrow j}, C_{i}\right)$ : Given a re-encryption key $r k_{i \leftrightarrow j}$ between $i$ and $j$ and a ciphertext $C_{i}$ for $i$, this algorithm outputs another ciphertext $C_{j}$ for $j$ or the error symbol $\perp . m \leftarrow \operatorname{Dec}(P P, s k, C)$ : Given a public key $s k$ and a ciphertext $C$, the decryption algorithm outputs a message $m$ or the error symbol $\perp$.

If the following two conditions holds, we say that the PRE scheme $\Pi$ satisfies correctness. For every $P P$ which is output from Setup $\left(1^{\lambda}\right)$, every $(p k, s k)$ which is output from KeyGen $(P P)$ and every message $m \in \mathcal{M}$, the probability $\operatorname{Pr}[C \leftarrow \operatorname{Enc}(P P, p k, m)$ : $\operatorname{Dec}(P P, s k, C)=m]$ is overwhelming. For every natural number $n \in \mathbb{N}$, every $P P$ which is output from Setup $\left(1^{\lambda}\right)$, every $\left(p k_{1}, s k_{1}\right) \ldots\left(p k_{n}, s k_{n}\right)$ which are outputs from KeyGen $(P P)$, every message $m \in M$, and every $r k_{1 \leftrightarrow 2} \ldots r k_{n-1 \leftrightarrow n}$ which are outputs from ReKeyGen $\left(r k_{i}, r k_{i+1}\right)$ for each $i \in[1, n-1]$, the probability $\operatorname{Pr}\left[C_{1} \leftarrow\right.$ $\operatorname{Enc}\left(P P, p k_{1}, m\right): \operatorname{Dec}\left(P P, s k_{n}, \operatorname{ReEnc}\left(P P, r k_{n-1 \leftrightarrow n}, \ldots \operatorname{ReEnc}\left(P P, r k_{1 \leftrightarrow 2}, C_{1}\right) \ldots\right)\right)=$ $m$ ] is overwhelming.

\subsection{Bidirectional and Multi-Hop PRE-CCA Security}

We prove that our scheme satisfies the PRE-CCA security in the full version of this paper. This security notion was proposed by Canetti and Hohenberger [6].

Definition 3 (Bidirectional and Multi-Hop PRE-CCA Security). Let $\lambda$ be the security parameter, $A$ be an oracle TM, representing the adversary, and $\Gamma_{U}$ and $\Gamma_{C}$ be date structures. Date structures $\Gamma_{U}$ and $\Gamma_{C}$ are first initialized as empty in the game. The game consists of an execution of $A$ with the following oracles, which can be invoked multiple times in any order, subject to the constraint below:

Setup Oracle: This oracle can be queried first in the game only once. This oracle makes a public parameter as $P P \leftarrow \operatorname{Setup}\left(1^{\lambda}\right)$. $A$ is given $P P$.

Uncorrupted key generation: This oracle generates a new key pair $(p k, s k) \leftarrow$ KeyGen $(P P)$ and adds $p k$ in $\Gamma_{U}$, where $P P$ is generated from the setup oracle. $A$ is given $p k$.

Corrupted key generation: This oracle generates a new key pair $(p k, s k) \leftarrow$ KeyGen $(P P)$ and adds $p k$ in $\Gamma_{C}$, where $P P$ is generated from the setup oracle. $A$ is given $(p k, s k)$.

Challenge oracle: This oracle can be queried only once. On input $\left(p k^{*}, m_{0}, m_{1}\right)$, the oracle chooses a bit $b \leftarrow\{0,1\}$ and returns $C^{*}=\operatorname{Enc}\left(P P, p k^{*}, m_{b}\right)$. We call $p k^{*}$ the challenge key and $C^{*}$ the challenge ciphertext. (We require the challenge key $p k^{*} \in \Gamma_{U}$ for $A$ to win.)

Re-encryption key generation: On input $\left(p k_{i}, p k_{j}\right)$ from the adversary, this oracle return the re-encryption key $r k_{i \leftrightarrow j}=\operatorname{ReKeyGen}\left(s k_{i}, s k_{j}\right)$, where $s k_{i}$ and $s k_{j}$ are the secret keys that correspond to $p k_{i}$ and $p k_{j}$, respectively. 
We require that $p k_{i}$ and $p k_{j}$ are in $\Gamma_{C}$, or alternatively both are in $\Gamma_{U}$. We do not allow for re-encryption key generation queries between a corrupted key and an uncorrupted key.

Re-encryption oracle: On input $\left(p k_{i}, p k_{j}, C_{i}\right)$, if $p k_{j} \in \Gamma_{C}$ and $\left(p k_{i}, C_{i}\right)$ is a derivative of $\left(p k^{*}, C^{*}\right)$, then return a special symbol $\perp$, which is not in the domain of messages or ciphertext. Else, return the re-encrypted ciphertext $C_{j}=$ $\operatorname{ReEnc}\left(\operatorname{ReKeyGen}\left(s k_{i}, s k_{j}\right), C_{j}\right)$. Derivatives of $\left(p k^{*}, C^{*}\right)$ are defined inductively as follows.

$-\left(p k^{*}, C^{*}\right)$ is a derivative of itself.

- If $(p k, C)$ is a derivative of $\left(p k^{*}, C^{*}\right)$, and $\left(p k^{\prime}, C^{\prime}\right)$ is a derivative of $(p k, C)$, then $\left(p k^{\prime}, C^{\prime}\right)$ is a derivative of $\left(p k^{*}, C^{*}\right)$.

- If $A$ has queried the re-encryption oracle on input $\left(p k, p k^{\prime}, C\right)$ and obtained response $C^{\prime}$, then $\left(p k^{\prime}, C^{\prime}\right)$ is a derivative of $(p k, C)$.

- If $A$ has queried the re-encryption key generation oracle on input ( $\left.p k, p k^{\prime}\right)$ or $\left(p k^{\prime}, p k\right)$, and $C^{\prime}=\operatorname{ReEnc}\left(\operatorname{ReKeyGen}\left(s k, s k^{\prime}\right), C\right)$, then $\left(p k^{\prime}, C^{\prime}\right)$ is a derivative of $(p k, C)$, where $s k$ and $s k^{\prime}$ are the secret keys that correspond to $p k$ and $p k^{\prime}$, respectively.

Decryption oracle: On input $(p k, C)$, if the pair $(p k, C)$ is a derivative of the challenge key and ciphertext $\left(p k^{*}, C^{*}\right)$, or $p k$ is not in $\Gamma_{U} \cup \Gamma_{C}$, then return a special symbol $\perp$ which is not in the domain of messages. Else, return $\operatorname{Dec}(s k, C)$, where $s k$ is the secret key that corresponds to $p k$.

Decision oracle: This oracle can be queried at the end of the game. On input $b^{\prime}$ : If $b^{\prime}=b$ and the challenge key $p k^{*} \in \Gamma_{U}$, then output 1 . Else, output 0 :

We describe the output of the decision oracle in the above game as $\operatorname{Expt}_{\Pi, A}^{\text {bid-PRE-CCA }}(\lambda)=$ $b$ for an adversary $A$ and a scheme $\Pi$. We define the advantage of adversary $A$ as

$$
\operatorname{Adv}_{I, A}^{\text {bid-PRE-CCA }}(\lambda) \stackrel{\text { def }}{=}\left|\operatorname{Pr}\left[\operatorname{Expt}_{\Pi, A}^{\text {bid-PRE-CCA }}(\lambda)=1\right]-\frac{1}{2}\right|
$$

where the probability is over the random choices of $A$ and oracles. We say that the scheme $\Pi$ is secure under the bidirectional PRE-CCA attack, if, for every adversary $A$, $\operatorname{Adv}_{\Pi, A}^{\text {bid-PRE-CCA }}(\lambda)$ is negligible in the security parameter $\lambda$.

\subsection{Description of Our Scheme}

We next describe our scheme. Let $\lambda$ be the security parameter, and let $n, k, k^{\prime}, k^{\prime \prime}$ and $v$ be parameters depending on $\lambda$. Let (SigGen, SigSign, SigVer) be a strongly unforgeable one-time signature scheme where verification keys are in $\{0,1\}^{v}$. Let (ParGen, LossyGen, LossyEval, Lossylnv, Relndex, ReEval, PrivReEval, Trans, FakeKey) be a collection of re-applicable $(n, k)$-LTDFs and $\mathcal{T}$ be a set of tags. Let $\left(\mathrm{G}_{\mathrm{abo}}, \mathrm{F}_{\mathrm{abo}}, \mathrm{F}_{\mathrm{abo}}^{-1}\right)$ be a collection of $\left(n, k^{\prime}\right)$-ABO trapdoor functions with branches $B_{\lambda}=\{0,1\}^{v}$, which contains the set of signature verification keys. Let $\mathcal{H}$ be a family of pairwise independent hash functions from $\{0,1\}^{n}$ to $\{0,1\}^{k^{\prime \prime}}$. We require that the above parameters are $\left(k+k^{\prime}\right)-\left(k^{\prime \prime}+n\right) \geq \delta=\delta_{1}+\delta_{2}$ for some $\delta_{1}=\omega(\log \lambda)$ and $\delta_{2}=\omega(\log \lambda)$. Our cryptosystem has message space $\{0,1\}^{k^{\prime \prime}}$. 
The algorithm Setup generates a public parameter $P P=\left(s_{\mathrm{abo}}, \mathrm{par}, h\right)$, and the algorithm KeyGen makes a pair of keys $(p k, s k)=\left(\left(s_{\text {rltdf }}, \tau\right),\left(t d_{\text {rltdf }}, s_{\text {rltdf }}, \tau\right)\right)$. Except a $\operatorname{tag} \tau$, we can consider that both Setup and KeyGen are the same algorithm as the key generation algorithm in the Peikert and Waters encryption. The algorithm Enc is also the same algorithm as the encryption algorithm in the Peikert and Waters encryption, except that $c_{1}$ is not signed for the re-encryption. The algorithm ReKeyGen makes a reencryption key, and the ReEnc re-encrypts a ciphertext into another ciphertext. These algorithms only use Relndex and ReEval in re-applicable LTDFs. The algorithm Dec is the same algorithm as the decryption algorithm in the Peikert and Waters encryption, expect that, if a ciphertext is re-encrypted, it applies PrivReEval for the validity check of ciphertexts.

$\operatorname{Setup}\left(1^{\lambda}\right)$ : $\operatorname{Setup}\left(1^{\lambda}\right)$ first generates an index of all-but-one trapdoor functions with lossy branch $0^{v}:\left(s_{\text {abo }}, t d_{\text {abo }}\right) \leftarrow \mathrm{G}_{\text {abo }}\left(1^{\lambda}, 0^{v}\right)$. Then, it generates a public parameter of re-applicable LTDFs: par $\leftarrow$ ParGen $\left(1^{\lambda}\right)$. Finally, it chooses a hash function $h \leftarrow \mathcal{H}$. It outputs a public parameter as $P P=\left(s_{\text {abo }}\right.$, par, $\left.h\right)$.

(The algorithm Setup erases the trapdoor $t d_{\text {abo }}$ because the following algorithms do not use $t d_{\text {abo. }}$ )

KeyGen $(P P)$ : KeyGen takes $P P=\left(s_{\text {abo }}\right.$, par, $\left.h\right)$ as input. It chooses a tag $\tau \in$ $\mathcal{T} \backslash\left\{\tau_{\text {los }}\right\}$ and generates an injective index of re-applicable LTDFs: $\left(s_{\text {rltdf }}, t d_{\text {rltdf }}\right) \leftarrow$ LossyGen $(\tau)$. A public key consists of the injective function index and the tag, and a secret key consisting of the trapdoor of $s_{\text {rltdf }}$ and the tag: $p k=\left(s_{\text {rltdf }}, \tau\right)$, and $s k=\left(t d_{\text {rltdf }}, s_{\text {rltdf }}, \tau\right)$.

$\operatorname{Enc}(P P, p k, m)$ : Enc takes $(P P, p k, m)$ as input, where $P P=\left(s_{\text {abo }}\right.$, par, $\left.h\right)$ is a tuple of public parameters, $p k=\left(s_{\text {rltdf }}, \tau\right)$ is a public key, and $m \in\{0,1\}^{\ell}$ is a message. It chooses $x \in\{0,1\}^{n}$ uniformly at random. It generates a key-pair for the one-time signature scheme: $\left(v k, s k_{\sigma}\right) \leftarrow \operatorname{SigGen}\left(1^{\lambda}\right)$, then computes

$$
c_{1}=\operatorname{LossyEval}\left(s_{\mathrm{rltdf}}, x\right), c_{2}=\mathrm{F}_{\mathrm{abo}}\left(s_{\mathrm{abo}}, v k, x\right), \text { and } c_{3}=h(x) \oplus m .
$$

Finally, it signs a tuple $\left(c_{2}, c_{3}, \tau\right)$ as $\sigma \leftarrow \operatorname{SigSign}\left(s k_{\sigma},\left(c_{2}, c_{3}, \tau\right)\right)$. Then, a ciphertext $C$ is output as $C=\left(v k, c_{1}, c_{2}, c_{3}, \tau, \sigma\right)$.

$\operatorname{ReKeyGen}\left(P P, s k_{i}, s k_{j}\right): \operatorname{ReKeyGen~takes~as~input~}\left(P P, s k_{i}, s k_{j}\right)$, where $\left(s k_{i}, s k_{j}\right)=$ $\left(\left(t d_{i}, s_{i}, \tau_{i}\right),\left(t d_{j}, s_{j}, \tau_{j}\right)\right)$. It computes $s_{i \leftrightarrow j} \leftarrow \operatorname{Relndex}\left(t d_{i}, t d_{j}\right)$, then outputs a reencryption key as $r k_{i \leftrightarrow j}=s_{i \leftrightarrow j}$.

$\operatorname{ReEnc}\left(P P, r k_{i \leftrightarrow j}, C_{i}\right):$ ReEnc takes $\left(r k_{i \leftrightarrow j}, C_{i}\right)$ as input, where $r k_{i \leftrightarrow j}=s_{i \leftrightarrow j}$ is a reencryption key and $C_{i}=\left(v k, c_{1, i}, c_{2}, c_{3}, \tau, \sigma\right)$ is a ciphertext. It computes $c_{1, j} \leftarrow$ $\operatorname{ReEval}\left(s_{i \leftrightarrow j}, c_{1, i}\right)$. It then outputs $C_{j}=\left(v k, c_{1, j}, c_{2}, c_{3}, \tau, \sigma\right)$ as a new ciphertext for the user with $s k_{j}$.

$\operatorname{Dec}(P P, s k, C)$ : Dec takes $(P P, s k, C)$ as input, where $P P=\left(s_{\text {abo }}\right.$, par, $\left.h\right)$ is a tuple of public parameters, $s k=\left(t d_{\mathrm{rltdf}}, s_{\mathrm{rltdf}}, \tau\right)$ is a secret key, and $C=\left(v k, c_{1}, c_{2}, c_{3}, \tau^{\prime}, \sigma\right)$ is a ciphertext. It first checks $\operatorname{SigVer}\left(v k,\left(c_{2}, c_{3}, \tau^{\prime}\right), \sigma\right)=1$; if not, it outputs $\perp$.

It then compute $x=\operatorname{Lossylnv}\left(t d_{\mathrm{rltdf}}, \tau^{\prime}, c_{1}\right)$. If $\tau=\tau^{\prime}$ then it checks $c_{1}=$ LossyEval $\left(s_{\text {rltdf }}, x\right)$, otherwise, it checks PrivReEval $\left(x, \tau^{\prime}, \tau, s_{\text {rltdf }}\right)=c_{1}$; if not, it outputs $\perp$. It also checks $c_{2}=\mathrm{F}_{\mathrm{abo}}\left(s_{\mathrm{abo}}, v k, x\right)$; if not, it outputs $\perp$. Finally, it outputs $m=c_{3} \oplus h(x)$. (We note that, if $C$ was not re-encrypted, then $\tau=\tau^{\prime}$. On the other hand, if $C$ was re-encrypted, $\tau \neq \tau^{\prime}$.) 


\subsection{Security of Our Scheme}

In this section, we claim the following theorem and describe a sketch of the proof. We give the detail proof in the full version of this paper.

Theorem 1. The above proposed scheme satisfies the PRE-CCA security.

We now show modifications of games from Game to $_{0} \mathrm{Game}_{10}$ to prove Theorem 1 $G_{a m e}$ is identical to the PRE-CCA game. In Game ${ }_{10}$, no adversary can win with meaningful probability. Every modification from $\mathrm{Game}_{i}$ to $\mathrm{Game}_{i+1}$ is perfect, statistically or computationally indistinguishable for each $i \in[0, n-1]$. Therefore, we conclude that no adversary also can win with meaningful probability in $\mathrm{Game}_{0}$.

In every game, let $C^{*}=\left(\nu k^{*}, c_{1}^{*}, c_{2}^{*}, c_{3}^{*}, \tau^{*}, \sigma^{*}\right)$ and $p k^{*}=\left(s_{\mathrm{rltdf}}^{*}, \tau^{*}\right)$ be the challenge ciphertext and the challenge public key, respectively.

Game $_{0}$ : This game is identical to the PRE-CCA game.

Game $_{1}$ : Let $x^{*}$ denote a random input applied to making the challenge ciphertext. (i.e. $c_{1}^{*}=\operatorname{LossyEval}\left(s_{\text {rltdf }}^{*}, x^{*}\right), c_{2}^{*}=\mathrm{F}_{\mathrm{abo}}\left(s_{\mathrm{abo}}, v k^{*}, x^{*}\right), c_{3}^{*}=h\left(x^{*}\right) \oplus m_{b}$. $)$

Then, we modify the decryption oracle as follows: The decryption oracle is given a decryption query $(p k, C)=\left((s, \tau),\left(v k, c_{1}, c_{2}, c_{3}, \tau^{\prime}, \sigma\right)\right)$, where $p k$ is limited to an output by the corrupted or the uncorrupted oracles. If $(p k, C)$ is a derivative of $\left(p k^{*}, C^{*}\right)$, then it outputs $\perp$. Else if the decryption query satisfies that $\left(v k, c_{2}, c_{3}, \tau^{\prime}, \sigma\right)=\left(v k^{*}, c_{2}^{*}, c_{3}^{*}, \tau^{*}, \sigma^{*}\right)$ and PrivReEval $\left(x^{*}, \tau^{\prime}, \tau, s\right)=c_{1}$, then it outputs $m \leftarrow c_{3} \oplus h\left(x^{*}\right)$. Otherwise, it outputs $\operatorname{Dec}(s k, C)$ in the ordinary decryption processes.

This modification does not affect any success probability of an adversary. From the injectivity of PrivReEval $\left(\cdot, \tau^{\prime}, \tau, s\right)$, if $\operatorname{PrivReEval}\left(x^{*}, \tau^{\prime}, \tau, s\right)=c_{1}$, then we obtain Lossylnv $\left(t d, \tau^{\prime}, c_{1}\right)=x^{*}$. In fact, the probability that the above queries satisfy $\operatorname{PrivReEval}\left(x^{*}, \tau^{\prime}, \tau, s\right)=c_{1}$ is negligible. We discuss this fact in the modification between Game $_{9}$ and Game $_{10}$. However, this check is necessary for the following modifications.

$G_{a m e}$ : We add the following check to the decryption oracle after checking a derivative: The decryption oracle is given a decryption query $(p k, C)=$ $\left((s, \tau),\left(v k, c_{1}, c_{2}, c_{3}, \tau^{\prime}, \sigma\right)\right)$. The decryption oracle always outputs $\perp$, if $v k=v k^{*}$ and $\left(c_{2}, c_{3}, \tau^{\prime}, \sigma\right) \neq\left(c_{2}^{*}, c_{3}^{*}, \tau^{*}, \sigma^{*}\right)$.

This modification is negligible for the success probability of an adversary from the strongly existential unforgeability of the signature scheme.

$\mathrm{Game}_{3}$ : Let $v k^{*}$ be the verification key used by the challenge oracle. We modify the setup oracle on a lossy branch as follows: We replace generates $\left(s_{\text {abo }}, t d_{\text {abo }}\right) \leftarrow$ $\mathrm{G}_{\text {abo }}\left(1^{\lambda}, 0^{v}\right)$ with $\left(s_{\mathrm{abo}}, t d_{\mathrm{abo}}\right) \leftarrow \mathrm{G}_{\mathrm{abo}}\left(1^{\lambda}, v k^{*}\right)$. The lossy branch is changed the verification key $v k^{*}$ from a zero-padding $0^{v}$.

This modification is negligible for the success probability of an adversary from the computational indistinguishability of all-but-one trapdoor functions. 
Game $_{4}$ : Let $\left(s_{\mathrm{abo}}, t d_{\mathrm{abo}}\right)$ be an index of all-but-one trapdoor functions and its trapdoor. We modify the decryption oracle as follows: The decryption oracle uses the trapdoor $t d_{\text {abo }}$ to decrypt ciphertext, when it receives a ciphertext $C=$ $\left(v k, c_{1}, c_{2}, c_{3}, \tau^{\prime}, \sigma\right)$. That is, in the case of $\left(v k, c_{2}, c_{3}, \tau^{\prime}, \sigma\right) \neq\left(v k^{*} c_{2}^{*}, c_{3}^{*}, \tau^{*}, \sigma^{*}\right)$ and $v k \neq v k^{*}$, we replace $x \leftarrow \operatorname{Lossylnv}\left(t d_{\mathrm{rltdf}}, \tau^{\prime}, c_{1}\right)$ with $x \leftarrow \mathrm{F}_{\mathrm{abo}}^{-1}\left(t d_{\mathrm{abo}}, v k, c_{2}\right)$, and proceed with the decryption processes (In the other cases, the decryption oracle executes the operations defined in Game ${ }_{1}$ and Game $_{2}$.).

This modification does not affect any success probability of an adversary because of the injectivity of LossyEval $(s, \cdot), \mathrm{F}_{\text {abo }}\left(s_{\text {abo }}, v k, \cdot\right)$, and PrivReEval $\left(\cdot, \tau^{\prime}, \tau, s\right)$. In this modification, $\mathrm{F}_{\mathrm{abo}}\left(s_{\mathrm{abo}}, v k, \cdot\right)$ is an injective function since $v k$ is not the lossy branch $v k^{*}$.

In the following games, the challenger manages uncorrupted re-encryption keys to apply the table. That is, in the first of this game, the challenger makes an empty table, which memorizes uncorrupted re-encryption keys. We set that a pair of keys $\left(p k_{1}, s k_{1}\right)$ is the first output by the uncorrupted oracle. At $n$-th query, the uncorrupted oracle outputs a pair of keys $\left(p k_{n}, s k_{n}\right)$ and makes the re-encryption keys $r k_{1 \leftrightarrow n}, \cdots, r k_{n-1 \leftrightarrow n}$ and $r k_{n \leftrightarrow 1}, \cdots, r k_{n \leftrightarrow n-1}$. The challenger adds re-encryption keys to the table.

Game $_{5}$ : We modify the re-encryption oracle as follows: The re-encryption oracle takes as query $\left(p k_{a}, p k_{b}, C_{a}\right)=\left(\left(s_{a}, \tau_{a}\right),\left(s_{b}, \tau_{b}\right),\left(v k, c_{1, a}, c_{2}, c_{3}, \tau_{a}^{\prime}, \sigma\right)\right)$ in the game. If $p k_{a}$ is corrupted, then it evaluates $x \leftarrow \operatorname{Lossylnv}\left(t d_{a}, \tau_{a}^{\prime}, c_{1, a}\right)$. Then, it makes $c_{1, b}=\operatorname{PrivReEval}\left(x, \tau_{a}, \tau_{b}, s_{b}\right)$. If $p k_{b}$ is uncorrupted, it searches $r k_{a \leftrightarrow b}$ in the reencryption keys table and evaluates $c_{1, b} \leftarrow \operatorname{ReEnc}\left(r k_{a \leftrightarrow b}, c_{1, a}\right)$. Then, it outputs $C_{b}=\left(v k, c_{1, b}, c_{2}, c_{3}, \tau_{a}^{\prime}, \sigma\right)$ as a re-encrypted ciphertext for $p k_{b}$.

This modification does not affect any success probability of an adversary because of the equivalence between $\operatorname{PrivReEval}\left(\cdot, \tau_{a}, \tau_{b}, s_{b}\right)$ and $\operatorname{ReEnc}\left(r k_{a \leftrightarrow b}, \operatorname{LossyEval}\left(s_{a}, \cdot\right)\right)$.

Game $_{6}$ : We modify the re-encryption key generation oracle as follows: Given a pair $\left(p k_{a}, p k_{b}\right)$, it searches the re-encryption keys $r k_{a \leftrightarrow b}$ from the table. Then, it outputs this re-encryption key $r k_{a \leftrightarrow b}$.

This modification does not affect any success probability of an adversary.

Game $_{7}:$ We define the number $q_{A \text {,unc }}$ as the maximum number of times that an adversary $A$ queries the uncorrupted oracle in the game. We modify the challenge oracle as follows.

First, the challenger chooses a random number $r \in\left\{1, \ldots, q_{A, \text { unc }}\right\}$. If the challenge oracle receives the challenge key $p k^{*} \neq p k_{r}$, the challenger outputs a random bit $b$ and aborts this game, where $p k_{r}$ is the $r$-th public key output by the uncorrupted oracle. Otherwise, it proceeds with this game.

This modification reduces the success probability of an adversary to $1 / q_{A \text {,unc }}$ fraction. However, this is not an important reduction since $q_{A \text {,unc }}$ is polynomial of the security parameter $\lambda$.

Game $_{8}$ : We modify the uncorrupted key generation oracle as follows.

First, it executes the following preprocessing. It choose a random number $r \in$ $\left\{1, \ldots, q_{A, \text { unc }}\right\}$ and generates a pair of keys $\left(p k_{r}, s k_{r}\right) \leftarrow \operatorname{KeyGen}(P P)$. We describe 
$p k_{r}=\left(s_{r}, \tau_{r}\right)$ and $s k_{r}=\left(t d_{r}, s_{r}, \tau_{r}\right)$. Then, for every $i \in\left\{1, \ldots, q_{A, \text { unc }}\right\} \backslash\{r\}$, it uses the fake key generation algorithm to generate a public key and the re-encryption key: it computes $\left(s_{i}, s_{r \leftrightarrow i}, \tau_{i}\right) \leftarrow \operatorname{FakeKey}\left(s_{r}, \tau_{r}\right)$ and sets $p k=\left(s_{i}, \tau_{i}\right), r k_{r \leftrightarrow i}$. Then, it computes that $r k_{i \leftrightarrow j}=s_{i \leftrightarrow j} \leftarrow \operatorname{Trans}\left(s_{r \leftrightarrow i}, s_{r \leftrightarrow j}\right)$ for every $i, j \in\left\{1, \ldots, q_{A, \text { unc }}\right\}$ and $i \neq j$. It adds every above-mentioned re-encryption key $\left\{r k_{i \leftrightarrow j}\right\}_{i, j \in\left\{1, \ldots, q_{A, \text { unc }}\right\}, i \neq j}$ to the re-encryption keys table.

At $j \in\left\{1, \ldots, q_{A, u n c}\right\}$ times query for the uncorrupted oracle, it outputs the public key $p k_{j}=s_{j}$ generated by the above preprocessing. The re-encryption key oracle and the re-encryption oracle execute their processes with the preprocessed table. The challenge oracle applies the above number $r$ to the check $p k^{*}=p k_{r}$.

This modification is negligible for the success probability of an adversary from the statistical indistinguishability of the fake key algorithm.

Game $_{9}$ : We modify the above preprocessing as follows: We replace the first key generation $p k_{r}=s_{r} \leftarrow \operatorname{LossyGen}\left(\tau_{r}\right)$ with $p k_{r}=s_{r} \leftarrow \operatorname{LossyGen}\left(\tau_{\text {los }}\right)$, where $\tau_{\text {los }}$ is a lossy tag.

This modification is negligible for the success probability of an adversary from the computational indistinguishability of LTDFs.

Game $_{10}$ : We modify the decryption oracle as follows: The decryption oracle always outputs $\perp$, when it receives the query $C=\left(v k^{*}, c_{1}, c_{2}^{*}, c_{3}^{*}, \sigma^{*}\right)$ and $p k=s_{\text {rltdf }}$.

This modification is negligible for the success probability of an adversary from the fact that the average-case min-entropy of $x^{*}$ is high, and every public key $p k=s$, except a challenge key, represents an injective function $f_{s, \star}$. That is, adversary never compute $c_{1}$ such that $f_{s, \star}^{-1}\left(c_{1}\right)=x^{*}$ information-theoretically. This fact implies that Game Ho $_{10}$ is statistically close to Game $_{9}$. Due to this modification, we attach tags to re-applicable LTDFs.

From the above sketch, we transform the PRE-CCA game (i.e. Game G $_{0}$ ) into the last game (i.e. Game ${ }_{10}$ ). In the last game, Game ${ }_{10}$, we conclude that any adversary does not detect which message is encrypted. The reason is, $h\left(x^{*}\right)$ is statistically close to $U_{\ell}$ since $x^{*}$ has high average-case min-entropy and $h(\cdot)$ can extracts a random string from $x^{*}$.

\section{Realization of Re-applicable LTDFs Based on DDH Assumption}

In this section, we describe the realization of re-applicable LTDFs from the DDH assumption. We modify the construction proposed by Peikert and Waters.

We now describe LTDFs based on the DDH assumption. We modify the construction as the proposed by Peikert and Waters on two points. One is a division of one function generation algorithm into two algorithms. The other is a change on an encrypting matrix in the injective function generator. Peikert and Waters proposed the function-generation algorithm which creates a function index as a ciphertext of a matrix. This ciphertext is encrypted with the ElGamal encryption on matrices. When generating an injectivefunction index, it encrypts the matrix $\boldsymbol{I}=\left(g^{\delta_{i, j}}\right)_{i, j}$ on $\mathbb{G}^{n \times n}$, where $\delta_{i, j}$ is the Kronecker delta. When generating a lossy-function index, it encrypts the matrix $\mathbf{0}=(e)_{i, j}$ on $\mathbb{G}^{n \times n}$, 
where $e$ is the identity in $\mathbb{G}$ (i.e. $e=g^{0}$ ). We also do the same for generating a lossyfunction index, but we do another procedure for generating an injective-function index. We define a set of tags $\mathcal{T}$ as a group $\mathbb{G}$ and a special element $\tau_{\text {los }}$ as the identity $e \in \mathbb{G}$. We use a matrix $\boldsymbol{M}=\left(\tau^{\delta_{i, j}}\right)_{i, j}$ on $\mathbb{G}^{n \times n}$, where $\tau$ is any element in $\mathbb{G}$. When generating a injective-function index, we set $\tau$ with $\tau \neq \tau_{\text {los }}$. When generating a lossy-function index, we set $\tau=\tau_{\text {los }}$.

- Generation of a public parameter. A parameter generator ParGen first executes $\mathcal{G}$, and $\mathcal{G}$ outputs a tuple $(p, \mathbb{G}, g)$. It next selects random numbers $r_{1}, \ldots, r_{n} \in_{R} \mathbb{Z}_{p}$, then makes a public parameter $\boldsymbol{C}_{1}$ as

$$
\boldsymbol{C}_{1}=\left(\begin{array}{c}
c_{1} \\
\vdots \\
c_{n}
\end{array}\right)=\left(\begin{array}{c}
g^{r_{1}} \\
\vdots \\
g^{r_{n}}
\end{array}\right)
$$

- Generation of function indices. A function generator LossyGen takes as input $\boldsymbol{C}_{1}$ and a $\operatorname{tag} \tau$, where $\boldsymbol{C}_{1}$ is the public parameter and $\tau$ is an element in $\mathbb{G}$. (We note that if $\tau=e$, it means execution of the lossy mode, otherwise, execution of the injective mode.) It first selects random elements $z_{1}, z_{2}, \ldots, z_{n} \in \in_{R} \mathbb{Z}_{p}$, then computes a function index as

$$
\boldsymbol{C}_{2}=\left(\begin{array}{ccc}
c_{1,1} & \cdots & c_{1, n} \\
\vdots & \ddots & \vdots \\
c_{n, 1} & \cdots & c_{n, n}
\end{array}\right)=\left(\begin{array}{ccc}
c_{1}^{z_{1}} \cdot \tau & \cdots & c_{1}^{z_{n}} \\
\vdots & \ddots & \vdots \\
c_{n}^{z_{1}} & \cdots & c_{n}^{z_{n}} \cdot \tau
\end{array}\right)=\left\{\begin{array}{cc}
c_{i, j}=c_{i}^{z_{j}} \cdot \tau & \text { if } i=j, \\
c_{i, j}=c_{i}^{z_{j}} & \text { otherwise }
\end{array}\right.
$$

The function index consists of $\left(\boldsymbol{C}_{1}, \boldsymbol{C}_{2}\right)$. A trapdoor consists of the random elements $z=\left(z_{1}, \ldots, z_{n}\right)$.

- Evaluation algorithm. An evaluation algorithm LossyEval takes as input $\left(\boldsymbol{C}_{1}, \boldsymbol{C}_{2}, \boldsymbol{x}\right)$, where $\left(\boldsymbol{C}_{1}, \boldsymbol{C}_{2}\right)$ is a function index, and $\boldsymbol{x}=\left(x_{1}, \ldots, x_{n}\right) \in\{0,1\}^{n}$ is an $n$-bit input interpreted as a vector.

It evaluates the linear product of $\boldsymbol{x}$ and $\boldsymbol{C}_{1}$ : That is, $y_{1}=\boldsymbol{x} \boldsymbol{C}_{1}=\prod_{i=1}^{n}\left(c_{i}\right)^{x_{i}}$.Next, it evaluates the product of $\boldsymbol{x}$ and $\boldsymbol{C}_{2}$ : That is, $\boldsymbol{y}_{2}=\boldsymbol{x} \boldsymbol{C}_{2}=\left(\prod_{i=1}^{n} c_{i, 1}^{x_{i}}, \cdots, \prod_{i=1}^{n} c_{i, n}^{x_{i}}\right)=$ $\left(\left(\prod_{i=1}^{n} c_{i}^{z_{1} x_{i}}\right) \tau^{x_{1}}, \cdots,\left(\prod_{i=1}^{n} c_{i}^{z_{n} x_{i}}\right) \tau^{x_{n}}\right)$. Finally, it outputs $\left(y_{1}, \boldsymbol{y}_{2}\right)$.

- Inversion algorithm. An inversion algorithm Lossylnv takes as input $\left(t d, \tau,\left(y_{1}, \boldsymbol{y}_{2}\right)\right)$, where trapdoor information $t d$ consists of $z=\left(z_{1}, \ldots, z_{n}\right), \tau$, which is an element in $\mathbb{G} \backslash\{e\}$, and $\boldsymbol{y}_{2}=\left(y_{2,1}, \cdots, y_{2, n}\right) \in \mathbb{G}^{1 \times n}$. It computes that $\boldsymbol{w}=\left(y_{2,1} \cdot y_{1}^{-z_{1}}, y_{2,2}\right.$. $\left.y_{1}^{-z_{2}}, \cdots, y_{2, n} \cdot y_{1}^{-z_{n}}\right)$. Then, if $j$-th element of $\boldsymbol{w}$ is the identity element of $\mathbb{G}$, then it sets $x_{j}=0$, else if $j$-th element of $w$ is $\tau$ then it sets $x_{j}=1$; otherwise, it output $\perp$. Finally, it outputs $x=\left(x_{1}, x_{2}, \ldots, x_{n}\right)$.

We can show that the above four algorithms satisfy injectivity, $(n, n-\log p)$-lossiness, and indistinguishability based on the DDH assumption. These proofs are nearly similar to the proof of Peikert and Waters [17]; therefore, we omit them in this paper.

Next, we show that the above algorithms satisfy the definition of re-applicable LTDFs. 
Re-applying with respect to function indices: Let $\tau_{i}$ and $\tau_{j}$ be tags different from $\tau_{\text {los }}$ in $\mathcal{T}$. Let $\left(s_{i}, t d_{i}\right)$ and $\left(s_{j}, t d_{j}\right)$ be outputs from LossyGen $\left(\tau_{i}\right)$ and LossyGen $\left(\tau_{j}\right)$. We now define an algorithm Relndex, which takes $t d_{i}$ and $t d_{j}$ as inputs and outputs $s_{i \leftrightarrow j}=t d_{j}-t d_{i}=\left(z_{1}^{\prime}-z_{1}, z_{2}^{\prime}-z_{2}, \ldots, z_{n}^{\prime}-z_{n}\right)=\left(z_{1, i \leftrightarrow j}, \ldots, z_{n, i \leftrightarrow j}\right)$.

We next define an algorithm ReEval. The algorithm ReEval takes as input $\left(s_{i \leftrightarrow j},\left(y_{1}, \boldsymbol{y}_{2}\right)\right)$, where $\left(y_{1}, \boldsymbol{y}_{2}\right)=\left(y_{1},\left(y_{2,1}, y_{2,2}, \cdots, y_{2, n}\right)\right)$ is an output from LossyEval $\left(s_{i}, \boldsymbol{x}\right)$. It computes that $\boldsymbol{y}_{2}^{\prime}=\left(y_{2,1}^{\prime}, y_{2,2}^{\prime}, \ldots, y_{2, n}^{\prime}\right)=\left(y_{2,1} \cdot y_{1}^{z_{1}, i \leftrightarrow j}, y_{2,2}\right.$. $\left.y_{1}^{z_{2, i \leftrightarrow j}}, \cdots, y_{2, n} \cdot y_{1}^{z_{n, i \leftrightarrow j}}\right)$. Then, it outputs $\left(y_{1}, \boldsymbol{y}_{2}^{\prime}\right)$.

For the above elements, we can describe that $\left(y_{1}, \boldsymbol{y}_{2}\right)=\left(g^{\sum_{i=1}^{n} x_{i} r_{i}},\left(g^{z_{1} \sum_{i=1}^{n} x_{i} r_{i}}\right.\right.$. $\left.\left.\tau_{i}^{x_{1}}, \cdots, g^{z_{n} \sum_{i=1}^{n} x_{i} r_{i}} \cdot \tau_{i}^{x_{n}}\right)\right)$ and $\left(y_{1}, \boldsymbol{y}_{2}^{\prime}\right)=\left(g^{\sum_{i=1}^{n} x_{i} r_{i}},\left(g^{z_{1}^{\prime} \sum_{i=1}^{n} x_{i} r_{i}} \cdot \tau_{i}^{x_{1}}, \cdots, g^{z_{n}^{\prime} \sum_{i=1}^{n} x_{i} r_{i}} \cdot \tau_{i}^{x_{n}}\right)\right)$. Therefore, we have $\boldsymbol{w}^{\prime}=\left(\tau_{i}^{x_{1}}, \cdots, \tau_{i}^{x_{n}}\right)$ in the algorithm Lossylnv $\left(t d_{j}, \tau_{i},\left(y_{1}, \boldsymbol{y}_{2}^{\prime}\right)\right)$. That is, we obtain that $\boldsymbol{x}=\operatorname{Lossylnv}\left(t d_{j}, \tau_{i},\left(y_{1}, \boldsymbol{y}_{2}^{\prime}\right)\right)$.

Generating proper outputs: Let $\tau_{i}, \tau_{j},\left(s_{i}, t d_{i}\right),\left(s_{j}, t d_{j}\right), s_{i \leftrightarrow j} \boldsymbol{x}$, and $\left(y_{1}, \boldsymbol{y}_{2}^{\prime}\right)$ be defined similarly to the above paragraph. We call $\left(y_{1}, \boldsymbol{y}_{2}^{\prime}\right)$ a proper output for $\boldsymbol{x}, \tau_{i}, \tau_{j}$, and $s_{j}$, if they satisfy $\left(y_{1}, \boldsymbol{y}_{2}^{\prime}\right)=\operatorname{ReEval}\left(s_{i \leftrightarrow j}, \operatorname{LossyEval}\left(s_{i}, \boldsymbol{x}\right)\right)$ for some $s_{i}$ made from a tag $\tau_{i}$ and $s_{i \leftarrow j}$. We can uniquely describe a proper $\left(y_{1}, \boldsymbol{y}_{2}^{\prime}\right)$ as $\left(g^{\sum_{i=1}^{n} x_{i} r_{i}},\left(g^{z_{1}^{\prime} \sum_{i=1}^{n} x_{i} r_{i}}\right.\right.$. $\left.\left.\tau_{i}^{x_{1}}, \cdots, g^{z_{n}^{\prime} \sum_{i=1}^{n} x_{i} r_{i}} \cdot \tau_{i}^{x_{n}}\right)\right)$ from the above algorithms, where $t d_{j}=\left(z_{1}^{\prime}, \cdots, z_{n}^{\prime}\right)$.

We define a new algorithm PrivReEval, which takes $x, \tau_{i}, \tau_{j}$, and $s_{j}$ as input, where $\boldsymbol{x}=\left(x_{1}, \cdots, x_{n}\right)$ is $n$-bits input. It computes $\left(\hat{y_{1}}, \hat{\boldsymbol{y}}_{2}\right) \leftarrow \operatorname{LossyEval}\left(s_{j}, \boldsymbol{x}\right)$. It makes $\hat{\boldsymbol{y}}_{2}^{\prime}$ from $\hat{\boldsymbol{y}}_{2}$ in the following process: for each $i \in[1, n]$, if $x_{i}=1$ then $\hat{y}_{2, i}^{\prime} \leftarrow \hat{y}_{2, i} \tau_{j}^{-1} \tau_{i}$, else $\hat{y}_{2, i} \leftarrow \hat{y}_{2, i}$, where $\hat{y}_{2, i}$ and $\hat{y}_{2, i}^{\prime}$ are the $i$-th elements of $\hat{\boldsymbol{y}}_{2}$ and $\hat{\boldsymbol{y}}_{2}^{\prime}$. Finally it outputs $\left(\hat{y}_{1}, \hat{\boldsymbol{y}}_{2}^{\prime}\right)$. The algorithm PrivReEval $\left(\boldsymbol{x}, \boldsymbol{\tau}_{i}, \boldsymbol{\tau}_{j}, s_{j}\right)$ always computes a proper output for $\boldsymbol{x}, \tau_{i}, \tau_{j}$, and $s_{j}$. The reason is that, from an output $\left(\hat{y}_{1}, \hat{\boldsymbol{y}}_{2}\right)=\left(g^{\sum_{i=1}^{n} x_{i} r_{i}},\left(g^{z_{1}^{\prime} \sum_{i=1}^{n} x_{i} r_{i}} \cdot \tau_{j}^{x_{1}}, \cdots, g^{z_{n}^{\prime} \sum_{i=1}^{n} x_{i} r_{i}} \cdot \tau_{j}^{x_{n}}\right)\right)$, we verify $\left(y_{1}, \hat{\boldsymbol{y}}_{2}^{\prime}\right)=$ $\left(g^{\sum_{i=1}^{n} x_{i} r_{i}},\left(g^{z_{1}^{\prime} \sum_{i=1}^{n} x_{i} r_{i}} \cdot \tau_{i}^{x_{1}}, \cdots, g^{z_{n}^{\prime} \sum_{i=1}^{n} x_{i} r_{i}} \cdot \tau_{i}^{x_{n}}\right)\right)$ that $\tau_{j}$ is replaced with $\tau_{i}$. This means that PrivReEval $\left(\cdot, \tau_{i}, \tau_{j}, s_{j}\right)$ is equivalent to $\operatorname{ReEval}\left(s_{i \leftrightarrow j}, \operatorname{LossyEval}\left(s_{i}, \cdot\right)\right)$ as a function.

Transitivity: We define an algorithm Trans, which takes $s_{i \leftrightarrow j}, s_{i \leftrightarrow k}$ and outputs $s_{i \leftrightarrow k}-$ $s_{i \leftrightarrow j}=\left(t d_{k}-t d_{i}\right)-\left(t d_{j}-t d_{i}\right)=t d_{k}-t d_{j}=s_{j \leftrightarrow k}$. That is, Trans $\left(s_{i \leftrightarrow j}, s_{i \leftrightarrow k}\right) \rightarrow s_{j \leftrightarrow k}$.

Statistical indistinguishability of the fake key: Now, we define an algorithm FakeKey, which takes a function index $s_{i}$ and a tag $\tau_{i}$ and makes a fake index $s_{j}$, a fake re-key $s_{i \leftrightarrow j}$, and a fake tag $\tau_{j}$. The fake key generator FakeKey takes as input $s_{i}=\left(\boldsymbol{C}_{1}, \boldsymbol{C}_{2}\right)$ and $\tau_{i} \in \mathbb{G}$, where $\left(\boldsymbol{C}_{1}, \boldsymbol{C}_{2}\right)$ is a function index. It then selects a random element $t \in \mathbb{G}$. It next chooses a random number $s_{i \leftrightarrow j}=\left(z_{1, i \leftrightarrow j}, \ldots, z_{n, i \leftrightarrow j}\right) \in_{R} \mathbb{Z}_{p}^{n}$, and makes a new matrix $\boldsymbol{C}_{2}^{\prime}$ as follows.

$$
\boldsymbol{C}_{2}^{\prime}=\left(\begin{array}{ccc}
c_{1,1} \cdot c_{1, i \leftrightarrow j}^{z_{1, i}} \cdot t \cdots & c_{1, n} \cdot c_{1}^{z_{n, i \leftrightarrow j}} \\
\vdots & \ddots & \vdots \\
c_{n, 1} \cdot c_{n}^{z_{1, i \leftrightarrow j}} & \cdots & c_{n, n} \cdot c_{n}^{z_{n, i \leftrightarrow j}} \cdot t
\end{array}\right)=\left\{\begin{array}{cc}
c_{k, \ell}^{\prime}=c_{k, \ell} \cdot c_{k}^{z_{l, i \leftrightarrow j}} \cdot t & \text { if } k=\ell, \\
c_{k, \ell}^{\prime}=c_{k, \ell} \cdot c_{k}^{z_{l, i \leftrightarrow j}} & \text { otherwise, }
\end{array}\right.
$$

where $c_{k}$ is the $k$ entry of $\boldsymbol{C}_{1}$, and $c_{k, l}$ is the $(k, l)$ entry of $\boldsymbol{C}_{2}$. Finally, it output $s_{j}=\left(\boldsymbol{C}_{1}, \boldsymbol{C}_{2}^{\prime}\right), s_{i \leftrightarrow j}=\left(z_{1, i \leftrightarrow j}, \ldots, z_{n, i \leftrightarrow j}\right)$, and $\tau_{j}=\tau_{i} \cdot t$.

From the abode description, outputs of FakeKey $\left(s_{i}, \tau_{i}\right)$ and the proper index have the same distribution. The reason is that, when $s_{i}$ and $\tau_{i}$ is made from the proper 
way, we can describe $\boldsymbol{C}_{2}^{\prime}$ by

$\boldsymbol{C}_{2}^{\prime}=\left(\begin{array}{ccc}g^{r_{1}\left(z_{1}+z_{1, i \leftrightarrow j}\right)} \cdot \tau_{i} \cdot t & \cdots & g^{r_{1}\left(z_{n}+z_{n, i \leftrightarrow j}\right)} \\ \vdots & \ddots & \vdots \\ g^{r_{n}\left(z_{1}+z_{1, i \leftrightarrow j}\right)} & \cdots & g^{r_{n}\left(z_{n}+z_{n, i \leftrightarrow j}\right)} \cdot \tau_{i} \cdot t\end{array}\right)=\left\{\begin{array}{cc}c_{k, \ell}^{\prime}=g^{r_{k}\left(z_{\ell}+z_{\ell, i \leftrightarrow j}\right)} \cdot \tau_{i} \cdot t & \text { if } k=\ell, \\ c_{k, \ell}^{\prime}=g^{r_{k}\left(z_{\ell}+z_{\ell, i \leftrightarrow j}\right)} & \text { otherwise, }\end{array}\right.$

where $\left(z_{1}, \cdots, z_{n}\right)$ is the trapdoor of $s_{i}$. This means that, conditioned on $t \neq\left(\tau_{i}\right)^{-1}$, the distribution of $\left\{\tau_{i}, \tau_{j},\left(s_{i}, t d_{j}\right),\left(s_{j}, t d_{j}\right), s_{i \leftrightarrow j}\right\}$ is identical to $\left\{\tau_{i}, \tau_{j}\right.$, LossyGen $\left(\tau_{i}\right)$, LossyGen $\left(\tau_{j}\right)$, Relndex $\left.\left(t d_{i}, t d_{j}\right)\right\}$. That is, distributions between them are statistically indistinguishable since the probability of $t=\left(\tau_{i}\right)^{-1}$ is $1 / p$.

Generation of injective functions from lossy functions: Next, we consider FakeKey which $s_{i}=\left(\boldsymbol{C}_{1}, \boldsymbol{C}_{2}\right)$ is output from LossyGen $\left(\tau_{\text {los }}\right)$. In this case, we can describe $\boldsymbol{C}_{2}^{\prime}$ as

$$
\boldsymbol{C}_{2}^{\prime}=\left(\begin{array}{ccc}
g^{r_{1}\left(z_{1}+z_{1, i \leftrightarrow j}\right)} \cdot t \cdots & g^{r_{1}\left(z_{n}+z_{n, i \leftrightarrow j}\right)} \\
\vdots & \ddots & \vdots \\
g^{r_{n}\left(z_{1}+z_{1, i \leftrightarrow j}\right)} & \cdots & g^{r_{n}\left(z_{n}+z_{n, i \leftrightarrow j}\right)} \cdots t
\end{array}\right),
$$

where $\left(z_{1}, \cdots, z_{n}\right)$ are logarithms between $\boldsymbol{C}_{1}$ and $\boldsymbol{C}_{2}$. Then, assuming that $t \neq e$, LossyEval $\left(s_{j}, \cdot\right)$ represents an injective function $f_{s_{j}, t}$. This probability is $1-1 / p$ which is overwhelming in the security parameter $\lambda$.

\section{References}

1. Ateniese, G., Benson, K., Hohenberger, S.: Key-Private Proxy Re-encryption. In: Fischlin, M. (ed.) RSA Conference 2009. LNCS, vol. 5473, pp. 279-294. Springer, Heidelberg (2009)

2. Ateniese, G., Fu, K., Green, M., Hohenberger, S.: Improved Proxy Re-Encryption Schemes with Applications to Secure Distributed Storage. In: Network and Distributed System Security Symposium, NDSS. The Internet Society (2005)

3. Bellare, M., Hofheinz, D., Yilek, S.: Possibility and Impossibility Results for Encryption and Commitment Secure under Selective Opening. In: Joux, A. (ed.) EUROCRYPT 2009. LNCS, vol. 5479, pp. 1-35. Springer, Heidelberg (2009)

4. Blaze, M., Bleumer, G., Strauss, M.: Divertible Protocols and Atomic Proxy Cryptography. In: Nyberg, K. (ed.) EUROCRYPT 1998. LNCS, vol. 1403, pp. 127-144. Springer, Heidelberg (1998)

5. Boldyreva, A., Fehr, S., O’Neill, A.: On Notions of Security for Deterministic Encryption, and Efficient Constructions without Random Oracles. In: Wagner, D. (ed.) CRYPTO 2008. LNCS, vol. 5157, pp. 335-359. Springer, Heidelberg (2008)

6. Canetti, R., Hohenberger, S.: Chosen-ciphertext secure proxy re-encryption. In: Ning, P., di Vimercati, S.D.C., Syverson, P.F. (eds.) ACM Conference on Computer and Communications Security, Alexandria, Virginia, USA, Octorber 2007, pp. 185-194. ACM, New York (2007)

7. Deng, R.H., Weng, J., Liu, S., Chen, K.: Chosen-Ciphertext Secure Proxy Re-encryption without Pairings. In: Franklin, M.K., Hui, L.C.K., Wong, D.S. (eds.) CANS 2008. LNCS, vol. 5339, pp. 1-17. Springer, Heidelberg (2008)

8. Freeman, D.M., Goldreich, O., Kiltz, E., Rosen, A., Segev, G.: More Constructions of Lossy and Correlation-Secure Trapdoor Functions. In: Nguyen, P.Q., Pointcheval, D. (eds.) PKC 2010. LNCS, vol. 6056, pp. 279-295. Springer, Heidelberg (2010) 
9. Hohenberger, S., Rothblum, G.N., Shelat, A., Vaikuntanathan, V.: Securely Obfuscating Reencryption. In: Vadhan, S.P. (ed.) TCC 2007. LNCS, vol. 4392, pp. 233-252. Springer, Heidelberg (2007)

10. Ivan, A., Dodis, Y.: Proxy Cryptography Revisited. In: NDSS, The Internet Society (2003)

11. Khurana, H., Heo, J., Pant, M.: From Proxy Encryption Primitives to a Deployable SecureMailing-List Solution. In: Ning, P., Qing, S., Li, N. (eds.) ICICS 2006. LNCS, vol. 4307, pp. 260-281. Springer, Heidelberg (2006)

12. Libert, B., Vergnaud, D.: Unidirectional Chosen-Ciphertext Secure Proxy Re-encryption. In: Cramer, R. (ed.) PKC 2008. LNCS, vol. 4939, pp. 360-379. Springer, Heidelberg (2008)

13. Mambo, M., Okamoto, E.: Proxy Cryptosystems: Delegation of the Power to Decrypt Ciphertexts. IEICE transactions on fundamentals of electronics, Communications and computer sciences 80(1), 54-63 (1997)

14. Mol, P., Yilek, S.: Chosen-Ciphertext Security from Slightly Lossy Trapdoor Functions. In: PKC (2010)

15. Naor, M., Yung, M.: Public-key Cryptosystems Provably Secure against Chosen Ciphertext Attacks. In: STOC, New Orleans, Louisiana, USA, May 1990, pp. 427-437. ACM, New York (1990)

16. Nishimaki, R., Fujisaki, E., Tanaka, K.: Efficient Non-interactive Universally Composable String-Commitment Schemes. In: Pieprzyk, J., Zhang, F. (eds.) ProvSec 2009. LNCS, vol. 5848, pp. 3-18. Springer, Heidelberg (2009)

17. Peikert, C., Waters, B.: Lossy Trapdoor Functions and Their Applications. In: Ladner, R.E., Dwork, C. (eds.) STOC, Victoria, British Columbia, Canada, May 2008, pp. 187-196. ACM, New York (2008)

18. Rosen, A., Segev, G.: Efficient Lossy Trapdoor Functions based on the Composite Residuosity Assumption. Cryptology ePrint Archive, Report 2008/134 (2008), http://eprint.iacr.org/

19. Rosen, A., Segev, G.: Chosen-Ciphertext Security via Correlated Products. In: Reingold, O. (ed.) Theory of Cryptography. LNCS, vol. 5444, pp. 419-436. Springer, Heidelberg (2009)

20. Shao, J., Cao, Z.: CCA-Secure Proxy Re-encryption without Pairings. In: Jarecki, S., Tsudik, G. (eds.) PKC 2009. LNCS, vol. 5443, pp. 357-376. Springer, Heidelberg (2009)

21. Taban, G., Cárdenas, A.A., Gligor, V.D.: Towards a secure and interoperable DRM architecture. In: Yung, M., Kurosawa, K., Safavi-Naini, R. (eds.) Digital Rights Management Workshop, pp. 69-78. ACM, New York (2006)

22. Weng, J., Chow, S.S., Yang, Y., Deng, R.H.: Efficient Unidirectional Proxy Re-Encryption. Cryptology ePrint Archive, Report 2009/189 (2009), http://eprint.iacr.org/

23. Zhang, X., Chen, M.-R., Li, X.: Comments on Shao-Cao's Unidirectional Proxy ReEncryption Scheme from PKC 2009. Cryptology ePrint Archive, Report 2009/344 (2009), http://eprint.iacr.org 